\title{
Interactions between task repetition and psychosocial factors
}

\author{
${ }^{\mathrm{a}}$ Michael W. Riley*, ${ }^{\mathrm{b}}$ Yu-Ting Hung, ${ }^{\mathrm{b}}$ Mao-Jiun Wang, ${ }^{\mathrm{b}}$ Yueh-Ling Lin, and ${ }^{\mathrm{a} J e s s i c a}$ C. Blunk \\ ${ }^{a}$ Industrial and Management Systems Engineering Department, University of Nebraska-Lincoln, W356 Nebraska \\ Hall, Lincoln, NE 68588, USA \\ ${ }^{b}$ Industrial Engineering and Engineering Management Department, National Tsing Hua University, Hsinchu, \\ Taiwan, 300 ROC
}

\begin{abstract}
Psychosocial factors, individual factors, workplace requirements, and workplace organizational factors have all been reported as being associated with the risk of musculoskeletal disorders [11]. Huang, Feuerstein, and Sauter [5] described the various concepts and models proposed to link occupational stress and work-related upper extremity disorders, as well as the difficulty in verifying the suggested linkages. Huang et al. [5] suggested that decomposing a complex model would be an appropriate method to begin the investigations of the proposed models. Task repetition is a workplace requirement that is considered a potential risk factor for cumulative trauma and its relationship to psychosocial factors was investigated. The study found only weak to no statistically significant relationship between task repetition and reported psychosocial factors of job stress in workers in Taiwan.
\end{abstract}

Keywords: repetition, psychosocial, musculoskeletal

\section{Summary}

The purpose of the study was to determine specific linkages between any psychosocial factor and the cumulative trauma risk factor of task repetition. The research involved an in-depth literature review and an analysis of the repetitive lifting tasks of 32 parcel delivery drivers and their psychosocial work stress [6].

Lee, Yeh, Chen, and Wang [7] reported the results of the prevalence and psychosocial risk factors of upper extremity musculoskeletal pain in over 17,000 subjects in industries in Taiwan. The psychosocial factors were grouped into the four constructs of (1) problems with job content, (2) poor physical working conditions, (3) problems of relationships, and (4) organizational problems. They also considered (a) comfort of the working environment and (b) satisfaction with the safety and hygiene of the job. 
The working environments included by Lee et al. [7] were manufacturing, service, administration, construction, and agriculture. This major study in Taiwan concluded that job stress due to job content, physical working conditions, and organizational problems, as well as dissatisfaction with safety and hygiene were statistically significant in association with developing upper extremity musculoskeletal pain.

In another study, Wang et al. [7] reported that pain in the neck and shoulder regions was associated with work organization and personal factors among garment workers in California, US. The linkage seems clear since repetition of tasks is statistically associated with musculoskeletal disorders, which cause pain. Therefore, repetition of tasks can be a logical contributor to musculoskeletal pain.

In 2010, Nieuwenhuijsen et al. [8] reported a comprehensive review of psychosocial work environments and stress-related disorders in The Netherlands. The authors reviewed 2,769 papers and found only seven that suggested high job demands, low co-worker support, low supervisor support, low procedural justice, low relational justice, and high effort- reward imbalance predicted incidence of stress related disorders. All of these seven papers used a version of the General Health Questionnaire. Only three of the seven studies had high job demands, which might include related repetitiveness as a predictor of job stress.

Bonde investigated repetitiveness causing stress symptoms in 2011 in Denmark [1]. The study followed two groups $(2,033$ subjects in repetitive jobs and 813 subjects in non-repetitive jobs) over three years. A baseline was established and the testing was repeated after the first, second, and third year. The Setterlind stress profile inventory was used to conclude that physically monotonous or repetitive work was not associated with the occurrence or subsequent development of stress symptoms.

Carayon, Smith, and Haims [3] concluded that work organization and psychosocial factors might lead to psychological stress and work-related musculoskeletal risks, but this does not preclude traditional risk factors such as repetition, force, and posture from being musculoskeletal injury risk factors. Their work is further described in the 2001 International Encyclopedia of Ergonomics and Human Factors.

The relationship mechanisms between psychosocial factors and work-related musculoskeletal disorders are poorly understood [9]. Research studies suggest perceptions of intensified workload, monotonous work, limited job control, low job clarity, and low social support are associated with various work-related musculoskeletal disorders.

Two major difficulties arise in the studies. The first is that psychosocial factors are measured at the individual level and physical factors at the group level and often by methods with limited precision and accuracy. Secondly, "objective" measures for psychosocial methods are difficult to develop but "objective" physical measures are typically available [9].

Psychosocial factors represent a wide variety of conditions and can be classified in three separate groups: (1) factors associated with the job and work environment, (2) factors associated with extra-work 
environment, and (3) characteristics of the individual workers. The job and work environment include conditions such as work organization, job content, organizational characteristics, interpersonal relationships, temporal aspects of work, financial aspects, and community aspects. Extra-work refers to roles outside of work. The individual characteristics are genetic factors, acquired aspects (education), and dispositional factors (personality traits) [9].

Possible explanations of the relationships between psychosocial factors and musculoskeletal disorders are: (1) psychosocial demands produce muscle tension and exacerbate task-related biomechanical strain, (2) psychosocial demands affect awareness and reporting of musculoskeletal symptoms and/or perceptions of their cause, (3) initial episodes of physical pain may trigger a chronic nervous system response with both physical and mental components and (4) changes in physical work situations may also change psychosocial demands. In the research, there has not been a standard way of assessing psychosocial factors and their relationships to musculoskeletal disorders [9]. Even the scales used have not been equivalent.

Intensified workload falls in the psychosocial factor domain of job and work environment. This would encompass repetition since job content is a part of job and work environment and repetitiveness is an aspect of job content. Relationships are complex. For example, if a questionnaire respondent has a psychosocial concern about job content is it due to physical factors or job repetitiveness? The psychosocial factor of intensified workload has typically been measured by some type of perceived time pressure. In the reported research, intensified workload has been the most consistently associated with upper extremity musculoskeletal disorders [9].

The association of upper extremity musculoskeletal disorders and psychosocial factors (intensified workload, monotonous work, and low levels of social support) is stronger for neck/shoulder disorders than hands/wrist disorders. The results may be skewed because studies of the neck/shoulder typically have more psychosocial variables considered. Most studies with extensive psychosocial factors were in office settings. The pathophysiologic processes resulting from adverse psychosocial and work organization factors may exert a greater effect on the neck/shoulder muscles [9].

Bongers et al. [2] concludes that firm judgments on the role of psychosocial factors in the etiology of upper extremity problems are not attainable due to the cross sectional nature of most studies. The review of literature showed some association between pain and psychosocial factors in Taiwan, difficulty in modeling the relationships between psychosocial factors and cumulative trauma risk factors, difficult in psychosocial factor identification, and the complexity of the relationships.

\section{Lifting study}

In 2010, Hung gathered research data about the work environment of a group of 32 of parcel delivery drivers in Taiwan [6]. The primary purpose of the 
Hung study was to assess lower back pain in parcel delivery drivers. Drivers, who loaded, delivered, and unloaded parcels, were given extensive medical evaluation to assess the condition of the spine and the amount of work done by the drivers was calculated through biomechanical analysis using video-taping and analysis of job tasks. The drivers also completed psychosocial stress questionnaires. From the data, task repetition was determined by the number parcels delivered and received.

The psychosocial domains extracted from the Quality of Life Questionnaire (items 1-5) and the Job Stress Questionnaire (items 6-10) were: (1) whole quality of life, (2) physical factor, (3) psychological factor, (4) social relationships, (5) environmental factors, (6) job dissatisfaction, (7) lack of autonomy, (8) lack of encouragement, (9) work load job stress and, (10) poor interpersonal relationships.

From the Pearson Correlation Coefficient seen in Table 1, there were no statistically significant $($ alpha $=.05)$ correlations between the measure of repetition (packages handled daily) and the ten measures of psychosocial factors. This data implies that for this particular type of repetitive task (manual material handling as a parcel delivery service in Taiwan), there is no association of psychosocial factor with the risk factor of repetition.
In the Job Stress Questionnaire, the five domains were: job dissatisfaction, lack of autonomy, lack of encouragement, job stress, and poor interpersonal relationships. Only two questions were generally related to repetition ("The work is too monotonous." and "Repetitive work is too much.") and integrated into the job stress domain. Thus, in the psychosocial questionnaires used for the package delivery drivers, repetitiveness was neither a psychosocial factor nor sub factor. The number of repetitions was a physical musculoskeletal risk factor. Thus, the repetitiveness of the task (lifting/loading/unloading) did not manifest itself as a psychosocial stressor in the research of 32 drivers.

Hung [6] calculated the correlations between a number of variables besides those described. For example, the degree of tiredness variable was correlated with four of the 10 psychosocial variables ("job dissatisfaction", "lack of autonomy", "work load stress" and "poor interpersonal relationships"), but degree of tiredness was not correlated with number of packages delivered per day. The number of packages handled was correlated with discomfort in the upper back and discomfort in the lower back. Discomfort in the lower back was correlated with the job stress psychosocial factor. 
Table 1

Selected values of correlation analysis of delivery drivers by Hung

\begin{tabular}{|c|c|c|c|c|c|c|c|c|c|c|c|}
\hline & \#/day & QOL & Phys & Psyc & Soc & Env & JobDis & LAut & LEnc & JStr & PoorInt \\
\hline \#/day & 1 & -.02 & .04 & -.09 & .03 & -.09 & -.15 & -.24 & -.16 & -.19 & -.01 \\
\hline
\end{tabular}

None of the correlations in Table 1 were statistically significant (alpha $=0.05$ ) where \#/day is the average total (delivered and received by a driver) number of parcels per day for a one year period, QOL=Quality of Life, Phys=Physical domain, Psyc=Psychological domain, Soc=Social domain, Env=Environmental domain, JobDis=Job Dissatisfaction, LAut=Lack of Autonomy, LEnc=Lack of Encouragement JStr=Job Stress, and PoorInt=Poor Interpersonal Relationships.

\section{Conclusion}

Although the research study of parcel delivery drivers did not show any statistically significant relationships between a measure of repetition and psychosocial factors, this does not preclude the possibility of weak relationships between the two. Funding for this research study was provided by the Fulbright U.S. Scholar Program.

\section{References}

[1] Bonde, J., Mikkelsen, S., Andersen, J., Fallentin, N., Baelum, J., Svendsen, S., Thomsen, J., Frost, P., Kaegaard, A. and the PRIM Health Study Group. Understanding Work Related Musculoskeletal pain: does repetitive work cause stress symptoms? Occu. Environ. Med. 62:41-48 (2005).

[2] Bongers, P., Kremer, A. and ter Laak, J. Are Psychosocial Factors, Risk Factors for Symptoms and Signs of the Shoulder, Elbow, or hand/Wrist?: A Review of Epidemiological Literature.

[3] Carayon, P, Smith, M. and Haims, M. Work Organization, Job Stress, and Work-Related Musculoskeletal Disorders,
Human Factors, Vol. 4, 1999.

[4] Carayon, P, Smith, M, and Haims, M. Work Organization and Psychosocial Work Factors: Definitions. International Encyclopedia of Ergonomics and Human Factors, 2001.

[5] Huang, G., Feuerstein, M. and Sauter, S. Occupational Stress and Work-Related Upper Extremity Disorders: Concepts and Models. Am. J. Ind. Med. 41:298-314 (2002)

[6] Hung, Y. The Investigation of Low Back Problems among Home Delivery Drivers. MS Thesis, National Tsing Hua University, 2010.

[7] H.Y. Lee, W.Y. Yeh, C.W. Chen and J.D. Wang, Prevalence and psychosocial risk factors of upper extremity musculoskeletal pain in industries of Taiwan: a nationwide study, J. Occup. Health, 47 (2005), pp. 311-318.

[8] Nieuwenhuijsen, K., Bruinvels and Frings-Dresen, M. Psychosocial work environment and stress-related disorders, a systematic review. Occu. Med. 60:277-286 (2010).

[9] National Institute for Occupational Safety and Health. Musculoskeletal Disorders and Workplace Factors: A Critical Review of Epidemiologic Evidence for WorkRelated Musculoskeletal Disorders of the Neck, Upper Extremity, and Low Back. Ed., Bernard, B. July (1997). 
[10] Wang, P.C. Follow-up of neck and shoulder pain among sewing machine operators: The Los Angeles garment study. Am. J. Ind. Med. 53: 352-60 (2007)

[11] W.S. Marras, R.G. Cutlip, S.E. Burt, and T.R. Waters, National occupational research agenda (NORA) future directions in occupational musculoskeletal disorders health research Applied Ergonomics 40 (2009), pp. 15-22. 